\title{
Grand Unification Concept with Bio-force as the Fifth Fundamental Force
}

\author{
Siva Prasad Kodukula \\ Independent Researcher, Visakhapatnam, India
}

Email address:

sivkod@gmail.com

\section{To cite this article:}

Siva Prasad Kodukula. Grand Unification Concept with Bio-force as the Fifth Fundamental Force. International Journal of High Energy Physics. Vol. 3, No. 3, 2016, pp. 18-24. doi: 10.11648/j.ijhep.20160303.11

Received: April 12, 2016; Accepted: April 20, 2016; Published: May 14, 2016

\begin{abstract}
All the four fundamental forces of nature have been explained in terms of space time. Coupling constant of grand unification has been defined by space time. The density of space time calculated by 'Siva's classical equation for space time' describes the nature of fundamental force. Thus for all fundamental forces, there exist a separate universe with a separate space time and a separate 'maximum signal velocity'. The transformation has been explained as an expansion of a single universe for which maximum signal velocity is 'velocity of light'. This transformation affects the concept of consciousness. Thus all other forms of space times explained as different forces of nature within the limitations of consciousness. Thus 'Bio-Force' defined as a form of space time like all other fundamental forces and considered as a fifth fundamental force. Consciousness is an affect of interactions of 'Bio-Force'. Coupling Constants for 'gravity' and 'Bio field' have been calculated by space time concept and concerned maximum signal velocities. For other three forces strong force, electromagnetic force and weak forces, the space time has been defined by their coupling constants. All these calculations have been done by one single equation $\mathrm{Vd}=\mathrm{K}$. With this equation, space time parameters for all fundamental forces have been explained. The conclusions described grand unification of all five natural forces in a different way which has been extended to 'Bio-Force', an important aspect of consciousness.
\end{abstract}

Keywords: Bio-force, Consciousness, Fundamental Force, Gravitation, Grand Unification, Space Time, Coupling Constants

\section{Introduction}

All the Grand Unification Theories (GUT) are aiming to unify all four fundamental forces ie. Strong, Electromagnetic, Weak and Gravitational interactions. The three gauge interactions of the Standard Model which define the electromagnetic, weak and strong interactions or forces, are merged into one single force. This unified interaction is characterized by one larger gauge symmetry and thus several force carriers, but one unified constant. Unifying gravity with the other three interactions would provide a theory of everything (TOE), rather than a GUT. Nevertheless, GUTs are often seen as an intermediate step towards a TOE. The unification of forces is possible due to the energy scale dependence of force coupling parameters in quantum field theory called renormalization group running, which allows parameters with vastly different values at usual energies to converge to a single value at a much higher energy scale. As of 2012, all GUT models which aim to be completely realistic are quite complicated, even compared to the Standard Model, because they need to introduce additional fields and interactions, or even additional dimensions of space. The main reason for this complexity lies in the difficulty of reproducing the observed fermions masses and mixing angles. Due to this difficulty and due to the lack of any observed effect of grand unification so far, there is no generally accepted GUT model [1]. In physics, a coupling constant or gauge coupling parameter is a number that determines the strength of the force exerted in an interaction [2].

A coupling constant (or an interaction constant) is a parameter in the field theory, which determines the relative strength of interaction between particles or fields. In the quantum field theory the coupling constants are associated with the vertices of the corresponding Feynman diagrams. Dimensionless parameters are used as coupling constants, as well as the quantities associated with them that characterize 
the interaction and have dimensions. The examples are the dimensionless 'fine structure constant' of electromagnetic interaction and the electric elementary charge, measured in coulombs [3].

However, in classical mechanics one usually makes these decisions directly by comparing forces. However there is no perfect Grand unification Theory which includes gravity is also a part of Unification. At the same time the problems like wave function collapse, measurement problem and involvement of consciousness demanding a different perspective of Grand Unification which describes an integrated model described by a single equation or concept. There are lot of papers describing these problems from Wigner to present Research $[4,5,6]$. At this stage involvement of 'Super theory of Relativity' and 'film theory of the universe' clearly defined the terms the observer and observation [7]. This concept emphasized the formation of Consciousness which is an affect of 'Bio-Force' which is just like other four fundamental forces. As per its conclusions, we cannot avoid the importance of 'consciousness' which is the main constituent that differentiates the 'living' and 'non-living' things of the universe. In order to incorporate 'consciousness' in to physics 'Super theory of Relativity' [7] has been introduced to physics. Special theory of Relativity explains the transformations between physical objects or frames where as 'Super Theory of Relativity' explains the interactions between living things and the physical universe by 'Film Theory of the Universe' [7]. As per this concept the universe in which we are living is a result of slide show of films changing in minute fraction of second. There is no link between these films. The events are prefixed programmed. Universal films are also similar to movie films. All the films are separate. But universe as a whole is a continuous flow of events. The continuity is due to consciousness which is a direct consequence of Bio-force. The concept and concerned calculations cleared that why the Bio-force is also a fundamental force of nature rather than the origination of consciousness described by 'Orch OR theory' of Hameroff and Penrose [6]. In the process it has been concluded that all the five fundamental forces of nature including gravity and 'Bio -Force' can be unified and can be expressed by space time. As gravity space time is our conventional four dimensional space time with maximum signal velocity ' $c$ ', Siva's gravity equation $\mathrm{Vd}=\mathrm{K}$ and Siva's constant ' $\mathrm{K}$ ' $[8,9]$ plays a crucial role in the derivation of space time parameters of other forces. The complete space time parameters for each field has been derived by a single equation $\mathrm{Vd}=\mathrm{K}$ by using 'Siva's classical equation for space time' [10]. If we know mass of a particle, we can calculate the space time density of its own mass by two equations of Siva's classical equations of space time.

Here the coupling constant has been defined as the ratio of total energy of space time of specified force to equivalent electromagnetic radiation with different plank constant specified to that particular force. It is nothing but comparison of quantum energies of two forces as described in presently accepted calculation of coupling constants. Here separate space time parameters such as density of space time, maximum signal velocity in that space time and plank constant associated to that space time have been described for each fundamental force.

Bio-force as the fifth fundamental force will influence the 'physics of consciousness' since it is an important aspect of 'consciousness'. Theoretical back ground of 'Physics of consciousness' may be helpful for the development of technologies related to 'Artificial Intelligence' and 'humanoid robotics'

\section{Discussion and Concept}

A Gravity space time is an affect of consciousness. Which can be interpreted by Siva's Gravity Equation Vd $=\mathrm{K}$. When $\mathrm{V}=\mathrm{c}$, the space time describes whole universe contains maximum signal velocity as 'velocity of light'. In the same way there exist different space times for each fundamental force which can be described by same equation $\mathrm{Vd}=\mathrm{K}$. But it contains different maximum signal velocity. This complete space time is a form of energy and can be interpreted in wave equation $E=h v$. As per classical mechanics the ratios of energies is coupling constant. If plank constant remains constant in both the cases, the frequency will be different. But if we keep frequency as constant the plank constant has to be changed. Thus a separate plank constant and separate energy will exist for each space time and frequency remains constant. Frequency will be constant since all the space times will satisfy $c=v \lambda$ where ' $c$ ' is constant and ' $\lambda$ ' is constant because ' $\mathrm{d}$ ' is constant when ' $\mathrm{V}$ ' is maximum signal velocity(we must have to consider maximum signal velocity to take whole space time in to consideration). Whatever may be space times, they will be converted to our physical parameters of our four dimensional universe by obeying $c=v \lambda$.

Thus coupling constant 'the ratios of energies of different space times' as interpreted as the ratio of ' $\mathrm{h} v$ ' and its total energy' where ' $v$ ' follows the equation $\mathrm{Vd}=\mathrm{K}$ where ' $\mathrm{V}$ ' is maximum signal velocity in that space time, ' $K$ ' is Siva's constant and $v=\mathrm{c} / \mathrm{d}$.

We know the maximum signal velocities for ' gravity space time' i.e velocity of light ' $c$ 'and for 'Bio-Force' (source of consciousness) ' $\sqrt{2} \mathrm{c}$ ' as per Super theory of relativity [7]. So we can calculate all the parameters of the space times. But for other forces we do not know the maximum signal velocities. So by reverse calculation without changing concept, we can make a generalized way of calculation by known coupling constants as per gauge theories. This will conclude the complete set of parameters of space times for each fundamental force. The results have been tabulated in Table 1. 
Table 1. Grand Unification Coupling Constants \& Space-time Parameters.

\begin{tabular}{|c|c|c|c|c|c|c|c|}
\hline S.no & Force & $\begin{array}{l}\text { Coupling } \\
\text { Constant }\end{array}$ & \multicolumn{3}{|c|}{ Space-time Parameter(SI units) } & $\begin{array}{l}\text { Siva's Grand Unification } \\
\text { Constant for all }\end{array}$ & $\begin{array}{l}\text { Equation for } \\
\text { space -time }\end{array}$ \\
\hline \multirow{4}{*}{1} & \multirow{4}{*}{ Strong Force } & \multirow{4}{*}{1} & Space-time Radius & $d$ & $6.2501096 \times 10^{62}$ & \multirow{20}{*}{$\begin{array}{l}\text { GUR Constant } \\
\tau=3.906387 \times 10^{125} \\
\text { GUD Constant } \\
\sigma=5.90765 \times 10^{-156} \\
\text { GUV Constant } \\
\omega=2.63079 \times 10^{-122} \\
\text { GUP Constant } \\
h_{\alpha}=h \alpha\end{array}$} & \multirow{20}{*}{$\begin{array}{l}d^{2}=\tau \alpha^{3} \\
\gamma \alpha^{4}=\sigma \\
V^{2} \alpha^{3}=\omega \\
h_{\alpha}=h \alpha\end{array}$} \\
\hline & & & Space-time Density & $\gamma$ & $5.90765 \times 10^{-156}$ & & \\
\hline & & & Signal velocity & $V$ & $1.62197 \times 10^{-61}$ & & \\
\hline & & & Plank Constant & $h_{\alpha}$ & $6.6261 \times 10^{-34}$ & & \\
\hline \multirow{4}{*}{2} & \multirow{4}{*}{$\begin{array}{l}\text { Electromagnetic } \\
\text { Force }\end{array}$} & & Space-time Radius & $d$ & $3.89768521 \times 10^{58}$ & & \\
\hline & & 1 & Space-time Density & $\gamma$ & $2.081119 \times 10^{-150}$ & & \\
\hline & & $\overline{137}$ & Signal velocity & $V$ & $2.6009017 \times 10^{-58}$ & & \\
\hline & & & Plank Constant & $h_{\alpha}$ & $6.6261 \times 10^{-34}$ & & \\
\hline \multirow{4}{*}{3} & \multirow{4}{*}{ Weak interaction } & \multirow{4}{*}{$10^{-6}$} & Space-time Radius & $d$ & $6.2501096 \times 10^{53}$ & & \\
\hline & & & Space-time Density & $\gamma$ & $5.90765 \times 10^{-132}$ & & \\
\hline & & & Signal velocity & $V$ & $1.62197 \times 10^{-52}$ & & \\
\hline & & & Plank Constant & $h_{\alpha}$ & $6.6261 \times 10^{-40}$ & & \\
\hline \multirow{4}{*}{4} & \multirow{4}{*}{ Gravitation } & \multirow{4}{*}{$6.64 \times 10^{-47}$} & Space-time Radius & $d$ & $3.38142 \times 10^{-7}$ & & \\
\hline & & & Space-time Density & $\gamma$ & $3.03985 \times 10^{29}$ & & \\
\hline & & & Signal velocity & $V$ & $2.998 \times 10^{8}$ & & \\
\hline & & & Plank Constant & $h_{\alpha}$ & $4.39945 \times 10^{-80}$ & & \\
\hline \multirow{4}{*}{5} & \multirow{4}{*}{$\begin{array}{l}\text { Bio-Force } \\
\text { (related to } \\
\text { interactions of } \\
\text { consciousness) }\end{array}$} & \multirow{4}{*}{$5.27 \times 10^{-47}$} & Space-time Radius & $d$ & $4.5372 \times 10^{39}$ & & \\
\hline & & & Space-time Density & $\gamma$ & $7.65976 \times 10^{29}$ & & \\
\hline & & & Signal velocity & $V$ & $4.2398 \times 10^{8}$ & & \\
\hline & & & Plank Constant & $h_{\alpha}$ & $3.491868 \times 10^{-80}$ & & \\
\hline
\end{tabular}

\section{Calculation}

\subsection{Space Time Parameters for Gravity}

Siva's classical Equations for space time are [10]

$$
\begin{aligned}
& m=7.065 \times 10^{12} \mathrm{~d}^{1 / 3} \\
& \gamma \mathrm{d}^{8 / 3}=1.687 \times 10^{12}
\end{aligned}
$$

Where ' $\mathrm{m}$ ' is mass of any body and ' $\mathrm{d}$ ' is its 'radius of its own space. $\gamma$ is its space time density.

Siva's gravity equation is $[8,9]$

$$
\mathrm{Vd}=\mathrm{K}
$$

Where ' $\mathrm{V}$ ' is velocity of the body existed due to force of gravity at a point at distance ' $\mathrm{d}$ ' from any observer. It is obvious that velocity of light i.e $2.997925 \times 10^{8} \mathrm{mt} / \mathrm{sec}$ is the maximum signal velocity in our conventional four dimensional space time. This space time contains a density also.

We can compare it with any mass density as explained in Siva's classical space time equations.

We have equations for quantum of energy

$$
\begin{aligned}
& \mathrm{E}=\mathrm{h} v \\
& \mathrm{E}=\mathrm{mc}^{2}
\end{aligned}
$$

As per Siva's gravity equation $\mathrm{Vd}=\mathrm{K}$

The ' $\mathrm{d}$ ' will be maximum when ' $\mathrm{V}=\mathrm{c}$ '. This is the stage of the universe contains a signal velocity ' $c$ ' and the space time density representing 'gravitational field'.
So the dia of the universe $d_{u g}=\frac{K}{c}$

We have Siva's constant

$$
\mathrm{K}=2.0275 \times 10^{2} \mathrm{sqmts} / \mathrm{sec}
$$

And velocity of light $\mathrm{c}=2.997925 \times 10^{8} \mathrm{mt} / \mathrm{sec}$

$$
\begin{aligned}
& \therefore \mathrm{d}_{\mathrm{ug}}=\frac{\mathrm{K}}{\mathrm{c}}=6.7631 \times 10^{-7} \mathrm{mts} \\
& \therefore \text { Its radius }=3.3816 \times 10^{-7} \mathrm{mts}
\end{aligned}
$$

This radius can be substituted in 'Siva's classical equation for space time.

We have 'Siva's classical Equation for space time (1) (2)

$$
\mathrm{m}=7.065 \times 10^{12} \mathrm{~d}^{1 / 3}
$$

Where ' $d$ ' is radius of space time material (let us consider it as space time fluid) of its mass ' $\mathrm{m}$ '

$$
\therefore \mathrm{m}=7.065 \times 10^{12} \mathrm{~d}^{1 / 3}=7.065 \times 10^{12} \mathrm{~d}^{1 / 3}
$$

$\therefore \mathrm{m}=4.9221 \times 10^{10}$ Kgs. $\left(\because \mathrm{d}=3.3816 \times 10^{-7} \mathrm{mts}\right)$

And space time density as per equation (2)

$$
\gamma=3.0395 \times 10^{29} \mathrm{Kg} / \mathrm{cum}
$$

We have equation (5)

$$
\mathrm{E}=m c^{2}
$$

If we substitute the value of ' $m$ ' in equation (5)

The energy of gravity field

$$
\mathrm{E}=4.4237 \times 10^{27} \mathrm{~J}
$$


For this universe there will be separate ' $h$ ' say ' $h$ ' so that all other entities like 'c', ' $\lambda$ ' and ' $v$ ' will be unchanged.

Let us suppose a coupling constant for gravitational force ' $C_{g}$ ' exists and will affect plank constant ' $h$ '

Now the Total Gravity Energy 'E' converted to a radiation of frequency ' $v$ '.

Then we can get a relation

$$
\begin{aligned}
& \frac{E}{v} \times C_{g}=h \\
& C_{g}=\frac{h v}{E}
\end{aligned}
$$

Now complete gravitational field converted in to electromagnetic field.

Let us find out the plank constant for gravity field say

' $\mathrm{h}_{\mathrm{g}}$ ' For gravity field

$$
\mathrm{E}_{\mathrm{g}}=\mathrm{h}_{\mathrm{g}} v
$$

For electromagnetic field

$$
\mathrm{E}_{\mathrm{p}}=\mathrm{hv}
$$

By definition Coupling constant is ratio of graviton energy to photon energy

$$
C_{g}=\frac{E_{g}}{E_{p}}=\frac{h_{g}}{h_{p}}
$$

We have equation (4)

$$
\begin{aligned}
& E=h v \\
& \text { And } c=v \lambda
\end{aligned}
$$

Here $\lambda$ is the wave length and can not be more than of the universe $d_{u g}$ i.e $6.7631 \times 10^{-7} \mathrm{mts}$.

When $\lambda=d_{\mathrm{ug}}$

$$
v=4.4327 \times 10^{14} \mathrm{hz}
$$

If we substitute values of $\mathrm{E}=4.4237 \times 10^{27} \mathrm{~J}$; Plank Constant $\mathrm{h}=6.6261 \times 10^{-34} \mathrm{~J} . \mathrm{sec}$; $\mathrm{C}_{\mathrm{g}}$

Frequency $v=4.4327 \times 10^{14} \mathrm{hz}$, we can get the value of

$$
\begin{aligned}
& C_{g}=\frac{h v}{E} \text { equation (7) } \\
& =6.63958 \times 10^{-47}
\end{aligned}
$$

We have Equation (10)

$$
\begin{gathered}
C_{g}=\frac{E_{g}}{E_{p}}=\frac{h_{g}}{h_{p}} \\
h_{g}=h C_{g} \\
\therefore h_{g}=h C_{g} \\
=4.39945 \times 10^{-80} \mathrm{~J} . \mathrm{sec}
\end{gathered}
$$

Thus for gravitational force can be interpreted by space time and its values are

1. Space time density $\gamma=3.0395 \times 10^{29} \mathrm{Kg} / \mathrm{cum}$

2. Maximum signal velocity is velocity of light i.e $\mathrm{c}=2.997925 \times 10^{8} \mathrm{mt} / \mathrm{sec}$

3. Plank constant $\mathrm{h}_{\mathrm{g}}=4.39945 \times 10^{-80} \mathrm{~J}$.sec

\subsection{Space Time Parameters for Bio-force}

As per super theory of Relativity [7], the observer will have two different frames of reference for an observation by living things because of consciousness. Consciousness is an affect of 'Bio-Force' attributed to living things. Physical universe will not exist if there is no link between two consecutive films of the universe. This link is originated by consciousness due to the maximum signal velocity of space time concerned to 'Bio-Force' which is more than the velocity of light.

Let us find out the space time and the related quantities with numerical values.

As per Siva's gravity equation (3) $\mathrm{Vd}=\mathrm{K}$

The ' $d$ ' will be maximum when ' $V=\sqrt{2} c$ '. This is the stage of the universe represents a space time with a signal velocity ' $\sqrt{2} c$ ' and the space time density representing 'Biofield' causing consciousness in living things.

If we substitute the value of ' $V$ ' as $\sqrt{2} \mathrm{c}$ in Equation (6)

Similar to above calculation

So the diameter of the universe $d_{u c}=\frac{K}{\sqrt{2} c}$

We have Siva's constant

$$
\mathrm{K}=2.0275 \times 10^{2} \text { sqmts } / \mathrm{sec}[8][9]
$$

And velocity of light $\mathrm{c}=2.997925 \times 10^{8} \mathrm{mt} / \mathrm{sec}$

$$
\begin{aligned}
& \therefore \mathrm{d}_{\mathrm{uc}}=\frac{\mathrm{K}}{\sqrt{2} \mathrm{c}}=4.7822 \times 10^{-7} \mathrm{mts} \\
& \therefore \text { Its radius }=2.3911 \times 10^{-7} \mathrm{mts}
\end{aligned}
$$

This radius can be substituted in 'Siva's classical equation for space time.

We have 'Siva's classical Equation for space time (1)

$$
m=7.065 \times 10^{12} \mathrm{~d}^{1 / 3}
$$

Where ' $d$ ' is radius of space time material (let us consider it as space time fluid) of its mass ' $m$ '

$$
\therefore \mathrm{m}=7.065 \times 10^{12} \mathrm{~d}^{1 / 3}=7.065 \times 10^{12} \mathrm{~d}^{1 / 3}
$$

$\therefore \mathrm{m}=4.3851 \times 10^{10}$ Kgs. $\left(\because \mathrm{d}=2.3911 \times 10^{-7} \mathrm{mts}\right)$

And space time density as per equation (2)

$$
\gamma=7.6593 \times 10^{29} \mathrm{Kg} / \mathrm{cum}
$$

The energy of consciousness field $\mathrm{E}=7.88226 \times 10^{27} \mathrm{~J}$ $\left(\because \mathrm{E}=2 \mathrm{mc}^{2}\right.$ since signal is $\sqrt{2} \mathrm{c}$ )

For this universe there will be separate ' $h$ ' say ' $h_{g}$ ' so that all other entities like 'c' $y$ and $v$ will be same

Let us suppose a coupling constant for consciousness force ' $\mathrm{C}_{\mathrm{c}}$ ' exists and will affect plank constant ' $\mathrm{h}$ ' 
Now the Total consciousness Energy ' $E$ ' converted to a radiation of frequency ' $v$ '.

Then we can get a relation similar to equation (7)

$$
\mathrm{C}_{\mathrm{c}}=\frac{\mathrm{h} v}{\mathrm{E}}
$$

Now, complete Bio-Field converted in to electromagnetic field.

Let us find out the plank constant for Bio-Field originating consciousness say ' $h_{c}$ '

For Bio-Field

$$
\mathrm{E}_{\mathrm{c}}=\mathrm{h}_{\mathrm{c}} v
$$

For electromagnetic field

$$
\mathrm{E}_{\mathrm{p}}=\mathrm{hv}
$$

By definition Coupling constant is ratio of consciousness energy (Bio-Force energy) to photon energy

$$
\mathrm{C}_{\mathrm{c}}=\frac{\mathrm{E}_{\mathrm{c}}}{\mathrm{E}_{\mathrm{p}}}=\frac{\mathrm{h}_{\mathrm{c}}}{\mathrm{h}_{\mathrm{p}}}
$$

We have

$$
\mathrm{E}=\mathrm{h} v
$$

And $\mathrm{c}=v \lambda$

Here $\lambda$ is the wave length and can not be more than that of the universe $\mathrm{d}_{\mathrm{uc}}$ i.e $4.7822 \times 10^{-7} \mathrm{mts}$.

When $\lambda=d_{\mathrm{uc}}$

$$
v=6.268924 \times 10^{14} \mathrm{hz}
$$

If we substitute values of $\mathrm{E}=7.88226 \times 10^{27} \mathrm{~J}$;

Plank Constant $\mathrm{h}=6.6261 \times 10^{-34} \mathrm{~J}$. sec;

Frequency $v=6.268924 \times 10^{14} \mathrm{hz}$ in equation (14), we can get the value of $\mathrm{C}_{\mathrm{c}}$

$$
\begin{gathered}
C_{c}=\frac{h v}{E} \text { equation (14) } \\
=5.26987 \times 10^{-47}
\end{gathered}
$$

We have equation (16)

$$
\begin{aligned}
\mathrm{C}_{\mathrm{c}} & =\frac{\mathrm{E}_{\mathrm{c}}}{\mathrm{E}_{\mathrm{p}}}=\frac{\mathrm{h}_{\mathrm{c}}}{\mathrm{h}_{\mathrm{p}}} \\
\mathrm{h}_{\mathrm{c}} & =\mathrm{hC}_{\mathrm{c}} \\
\therefore \mathrm{h}_{\mathrm{c}} & =\mathrm{hC}_{\mathrm{c}} \\
& =3.49187 \times 10^{-80} \mathrm{~J} . \mathrm{sec}
\end{aligned}
$$

Thus for gravitational force can be interpreted by space time and its values are

1. Space time density $\gamma=7.6593 \times 10^{29} \mathrm{Kg} / \mathrm{cum}$

2. Maximum signal velocity is $\sqrt{2}$ times velocity of light i.e $4.239706194 \times 10^{8} \mathrm{mts} / \mathrm{sec} / \mathrm{sec}$

3. Plank constant $=3.49187 \times 10^{-80} \mathrm{~J} . \mathrm{sec}$

\section{Generalized Equation for Space-Time Parameters}

In the above two cases i.e. gravity and 'Bio-field', we have signal velocity. We have calculated the coupling constant and space time density. But in the case of other three forces i.e. electromagnetic, week interactions and strong force, we do not know the signal velocity. So let us back calculate the space time density and signal velocities by considering the coupling constants for those three forces derived by other means [11].

For convenience let us generalize the calculations

\subsection{Relation Between Coupling Constant and Space-Time Radius}

Let us suppose the coupling constant ' $\alpha$ ' and maximum signal velocity ' $\mathrm{V}$ '

We can say $\alpha=\frac{\mathrm{h} v}{\mathrm{E}}$ Similar to Equations (7)(14)

Where ' $E$ ' is the total energy of space time of the specified fundamental force.

$$
\begin{gathered}
\therefore \mathrm{E}=\frac{\mathrm{h} v}{\alpha} \\
\therefore \mathrm{mV}^{2}=\frac{\mathrm{h} v}{\alpha}
\end{gathered}
$$

$\left(\because \mathrm{E}=\mathrm{mV}^{2}\right.$ Where ' $\mathrm{V}$ ' is maximum signal velocity in the 'space time with a coupling constant $\alpha^{\text {') }}$

We have Siva's Classical Equation for space time (1)

$$
\mathrm{m}=7.065 \times 10^{12} \mathrm{~d}^{1 / 3}
$$

Where ' $d$ ' is radius of space time of mass ' $m$ '

And As per Siva's gravity equation (3) $\mathrm{Vd}=\mathrm{K}$

This is the stage of the universe represents a universe as explained in 'Heart of God' model of the universe contains a signal velocity ' $\mathrm{V}$ ' and the space time density.

So the dia of the universe $d_{u}=\frac{K}{V}$

Where Siva's constant $K=2.0275 \times 10^{2}$ sqmts/sec

$$
\therefore \text { Radius ' } \mathrm{d}^{\prime}=\frac{\mathrm{K}}{2 \mathrm{~V}}
$$

This radius can be substituted in 'Siva's classical equation for space time.

This can be rewrite as

' $\mathrm{V}^{\prime}=\frac{\mathrm{K}}{2 \mathrm{~d}}$ Where ' $\mathrm{V}$ ' is maximum signal velocity at this stage of Universe.

$$
\begin{gathered}
\therefore \mathrm{mV}^{2}=\frac{\mathrm{h} v}{\alpha} \text { equation (18) } \\
\therefore 7.065 \times 10^{12} \mathrm{~d}^{1 / 3} \times\left(\frac{\mathrm{K}}{2 \mathrm{~d}}\right)^{2}=\frac{\mathrm{h} v}{\alpha} \\
\therefore \frac{\mathrm{d}^{2}}{\mathrm{~d}^{1 / 3}}=\frac{7.065 \times 10^{2} \mathrm{~K}^{2}}{4 \times \mathrm{h} v} \times(\alpha)
\end{gathered}
$$

We have electromagnetic wave equation $c=v \lambda$ $\lambda$ is maximum When $\lambda=\mathrm{d}_{\mathrm{u}}$ 


$$
\begin{aligned}
& \therefore v=\frac{\mathrm{c}}{\mathrm{d}_{\mathrm{u}}} \quad\left(\because \mathrm{c}=v \lambda \text { and } \lambda=\mathrm{d}_{\mathrm{u}}\right) \\
& \therefore v=\frac{\mathrm{c}}{2 \mathrm{~d}} \quad\left(\because \mathrm{d}_{\mathrm{u}}=2 \mathrm{~d}\right)
\end{aligned}
$$

If we substitute the values of $\mathrm{K}=2.0275 \times 10^{2}$ sqmts / sec and ' $v$ ' and ' $h$ ' and 'c' in equan. (21)

$$
\begin{gathered}
\therefore \frac{\mathrm{d}^{2}}{\mathrm{~d}^{1 / 3}}=\frac{7.065 \times 10^{12} \mathrm{~K}^{2}}{4 \times \mathrm{h} v} \times(\alpha) \\
\therefore \frac{\mathrm{d}^{2}}{\mathrm{~d}^{1 / 3}}=\frac{7.065 \times 10^{12} \times\left(2.0275 \times 10^{2}\right)^{2}}{4 \times 6.6261 \times 10^{-34} \times\left(\frac{\mathrm{c}}{2 \mathrm{~d}}\right)} \times(\alpha) \\
\therefore \frac{\mathrm{d}}{\mathrm{d}^{1 / 3}}=\frac{7.065 \times 10^{12} \times\left(2.0275 \times 10^{2}\right)^{2}}{2 \times 6.6261 \times 10^{-34} \times 2.997925 \times 10^{8}} \times(\alpha) \\
\therefore \frac{\mathrm{d}}{\mathrm{d}^{1 / 3}}=\frac{7.065 \times 10^{12} \times\left(2.0275 \times 10^{2}\right)^{2}}{2 \times 6.6261 \times 10^{-34} \times 2.997925 \times 10^{8}} \times(\alpha) \\
\therefore \mathrm{d}^{2 / 3}=7.31013 \times 10^{41} \times \alpha \\
\therefore \mathrm{d}^{2}=390.63873 \times 10^{123} \times \alpha^{3} \\
\therefore \mathrm{d}^{2}=3.906387 \times 10^{125} \times \alpha^{3}
\end{gathered}
$$

Let us Say $\tau=3.906387 \times 10^{125}$

Therefore final Equation is

$$
\mathrm{d}^{2}=\tau \alpha^{3}
$$

Where $^{\prime} \tau^{\prime}=3.906387 \times 10^{125}$

$$
d^{2}=\tau \alpha^{3}
$$

Where

$d$ is space time radius

$\alpha$ is coupling constant for it

$\tau$ is Siva's GUR constant

\subsection{Relation Between Coupling Constant and Space Time Density}

We have Siva's classical Equation for space time in terms of its density (2) is

$$
\begin{gathered}
\gamma \mathrm{d}^{8 / 3}=1.687 \times 10^{12} \\
\gamma\left(3.906387 \times 10^{125} \times \alpha^{3}\right)^{4 / 3}=1.687 \times 10^{12} \\
\left(\text { since equation }(24) \mathrm{d}^{2}=3.906387 \times 10^{125} \times \alpha^{3}\right) \\
\gamma\left(390.6387 \times 10^{123} \times \alpha^{3}\right)^{4 / 3}=1.687 \times 10^{12} \\
\gamma \alpha^{4}=\frac{1.687 \times 10^{12}}{2855.6196 \times 10^{164}} \\
\gamma \alpha^{4}=5.90765 \times 10^{-156} \\
\text { Say } \sigma=5.90765 \times 10^{-156} \\
\gamma \alpha^{4}=\sigma \\
\gamma \alpha^{4}=\sigma
\end{gathered}
$$

Where $\gamma$ is space time density

$\alpha$ is coupling constant for space time

\subsection{Relation Between Coupling Constant and Maximum Signal Velocity in That Space Time}

We have Equation (20)

$$
\begin{gathered}
{ }^{\prime} \mathrm{V}^{\prime}=\frac{\mathrm{K}}{2 \mathrm{~d}} \\
\therefore \mathrm{V}^{2}=\frac{\mathrm{K}^{2}}{4 \mathrm{~d}^{2}} \\
\therefore \mathrm{V}^{2}=\frac{\mathrm{K}^{2}}{4 \times 3.906387 \times 10^{125} \times \alpha^{3}}
\end{gathered}
$$

$\left(\because\right.$ Equation $(24)$ is $\left.\mathrm{d}^{2}=3.906387 \times 10^{125} \times \alpha^{3}\right)$

$$
\begin{aligned}
\therefore \mathrm{V}^{2} \alpha^{3} & =\frac{\left(2.0275 \times 10^{2}\right)^{2}}{4 \times 3.906387 \times 10^{125}} \\
\therefore \mathrm{V}^{2} \alpha^{3} & =2.63079 \times 10^{-122}
\end{aligned}
$$

Let us say $\omega=2.63079 \times 10^{-122}$

$$
\begin{aligned}
\therefore \mathrm{V}^{2} \alpha^{3} & =\omega \\
V^{2} \alpha^{3} & =\omega
\end{aligned}
$$

Where

$V$ is maximum signal velocity

$\alpha$ is coupling constant for space time

\subsection{Relation Between Coupling Constant and PLANK Constant in That Space Time}

We have equation (12)

$$
\mathrm{h}_{\mathrm{g}}=\mathrm{hC}_{\mathrm{g}}
$$

If we generalize the equation for all natural forces We can rewrite it as

$$
\mathrm{h}_{\alpha}=\mathrm{h} \alpha
$$

Where ' $\alpha$ ' is coupling constant for concerned space time and ' $h$ ' is plank constant and ' $h{ }_{\alpha}$ ' is plank constant for concerned space time.

$$
h_{\alpha}=h \alpha
$$

Where

$\mathrm{h}$ is Plank constant $\alpha$ is coupling constant for space time $h_{\alpha}$ is Siva's GUP constant

\section{Calculation of 'Space-Time Parameters' by Generalized Equations}

With the above generalized equations, we can find Grand Unification Values for all the fundamental forces. 
i) Grand Unification Constant for Radius(GUR)

ii) Grand Unification Constant for Density(GUD)

iii) Grand Unification Constant for Maximum

iv) Signal Velocity (GUV)

v) Grand Unification Constant for Plank constant(GUP)

Here coupling constant for 'Gravity' and 'Bio-Force' have been considered from this concept since we know the maximum signal velocities for those space times. But for 'Strong force', 'Electromagnetic force' and 'Weak Interactions' we do not know the space times and maximum signal velocity associated to it. So for these three forces the space time parameters have been calculated by considering the coupling constant provided by other works [11]. The results have been tabulated in Table 1 .

\section{Conclusions}

1. Consciousness is an affect of Bio-field which contains bio force and is similar to Fundamental forces of universe like Strong force, Electromagnetic force, weak force and Gravity force.

2. All the fundamental forces including Gravity and bio-force will be explained by their space time parameters.

3. Space time parameters for all five fundamental forces has been interpreted by one single equation $\mathrm{Vd}=\mathrm{K}$

4. Space time Parameters for all fundamental forces are

i. Maximum signal velocity is ' $\mathrm{V}$ '

ii. Space time radius ' $d$ '

iii. Space time density' $\gamma$ '

iv. Plank constant for that space time ' $h_{\alpha}$ '

v. Coupling constant ' $\alpha$ '

5. Space time Equations for all five fundamental forces are

i. $V^{2} \alpha^{3}=\omega$

ii. $\mathrm{d}^{2}=\tau \alpha^{3}$

iii. $\gamma \alpha^{4}=\sigma$

iv. $\mathrm{h}_{\alpha}=\mathrm{h} \alpha$

6. Siva's Grand Unification Constants.

i. Grand Unification Constant for Radius (GUR) i.e $\tau=3.91 \times 10^{125} \mathrm{mts}$.

ii. Grand Unification Constant for Density (GUD) i.e $\sigma=5.90765 \times 10^{-156} \mathrm{Kg} . \mathrm{m}^{-3}$

iii. Grand Unification Constant for maximum signal Velocity (GUV) $\omega=2.63079 \times 10^{-122} \mathrm{~m} \cdot \mathrm{sec}^{-1}$

iv. Grand Unification Constant for Plank constant (GUP) $\mathrm{h}_{\alpha}$ is depending upon coupling constant and is equal to ' $h \alpha$ '. Where ' $h$ ' is plank constant and ' $\alpha$ ' is coupling constant.

7. All the results have been tabulated in Table 1.

\section{References}

[1] https://en.wikipedia.org/wiki/Grand_Unified_Theory.

[2] https://en.wikipedia.org/wiki/Coupling_constant.

[3] https://en.wikiversity.org/wiki/Coupling_constant.

[4] R. W. Boyer, "Unpacking Quantum Wave Function Collapse", Neuroquantology, Vol. 10, No. 2 (2012)230-251. http://www.neuroquantology.com/index.php/journal/article/vie $\mathrm{w} / 530$

[5] Esfeld, M., "Essay Review Wigner's View of Physical Reality", Studies in History and Philosophy of Modern Physics, Vol. 30B (1999) 145-154.

http://www.unil.ch/files/live//sites/philo/files/shared/DocsPers o/EsfeldMichael/1999/SHPMP99.pdf

[6] R. Penrose, S. Hameroff, "Consciousness in the Universe: Neuroscience, Quantum Space-time Geometry and Orch OR Theory", Journal of Cosmology, Vol. 14(2011).

http://journalofcosmology.com/Consciousness $160 . \mathrm{html}$.

[7] S. P. Kodukula, "Super Theory of Relativity-Explanation to 'Rest Mass of Photon', 'Quantum Entanglement' and 'Consciousness'", American Journal of Modern Physics, Vol. 3, No. 6(2014). 232-239.

http://article.sciencepublishinggroup.com/pdf/10.11648.j.ajmp .20140306.15.pdf

[8] S. P. Kodukula, "New Cosmological Cons tants", FFP9 (Conference 2008 Udine, Italy.

http://www.fisica.uniud.it/ deangeli/test/AbstractsFFP9.pdf

[9] S. P. Kodukula, "Derivation of Siva's Constant 'K' of Physics", IJoART, Vol. 2, No. 1 (2013).

http://www.ijoart.org/docs/Derivation-of-Sivas-Constant-K-of -Physics.pdf

[10] S. P. Kodukula, "Siva's Classical Equation for Space Time and Matter”, IJoART, Vol. 2, No. 8 (2013).

http://www.ijoart.org/docs/Sivas-Classical-Equation-for-Space -Time-and-Matter.pdf

[11] C. R. Nave. C, "Hyper physics", Georgia state university (2012).

http://hyperphysics.phy-astr.gsu.edu/hbase/forces/couple.html $\# \mathrm{c} 1$. 